\title{
The influence of urban form on spatial costs
}

\author{
Hugh B. Wenban-Smith *
}

\section{Introduction}

There is a general presumption in urban economics that average commuting costs are increasing in city size (e.g. Abdel-Rahman \& Anas (2004), pp. 2298-2300). An early statement of this result can be found in Arnott (1979). Arnott shows average commuting cost to be an increasing function of city size by considering a circular city of uniform population density, where commuters travel individually and radially to a central business district and transport cost is proportional to distance. Aggregate commuting costs are then given by:

$$
A C C=\int_{0}^{R} t \cdot \bar{d} \cdot r \cdot 2 \pi r \cdot d r=t \cdot \bar{d} \cdot \frac{2}{3} \pi \cdot R^{3}=\frac{2 t}{3 \sqrt{\pi \cdot \bar{d}}} P^{3 / 2}
$$

Where $\bar{d}$ is the population density, $R$ is the radius of the city, $P$ is its population and $t$ is unit transport cost, i.e. aggregate commuting costs increase more than proportionately with population, and average commuting cost $(A C C / P)$ is an increasing function of $P$ - and hence to be seen as a negative influence on agglomeration.

By analogy, it might be supposed that other spatial costs, such as distribution costs for utility services or access costs to facilities such as schools and hospitals, will have the same characteristic. However, it is more realistic to suppose that population density declines away from the centre, as in the standard monocentric urban model (Brueckner (1982), Fujita (1989, Chs 3\&4), Fujita \& Thisse (2002, pp. 78-83)) and that there are likely to be scale economies in both commuting (e.g. from use of buses or metros) and distribution (e.g. larger diameter water mains). The aim of this paper is to explore the implications of such factors for spatial costs. Of particular

Department of Geography \& Environment, London School of Economics. (E-mail: wenban @globalnet.co.uk ) 
interest is the question whether there are circumstances in which average spatial costs may be decreasing in city size, and so favour agglomeration, contrary to the usual presumption.

The case of water supply has not previously been used to investigate this question, but people moving to and from the centre along fixed routes (whether in cars, buses or tube trains) are not very different conceptually from water moving from a treatment works through pipes to people's homes. Both require investment in a connecting network, the economics of which are affected by the numbers and location of customers, and may involve a trade-off between economies of scale in production and spatial costs.

The structure of the paper is as follows:

- First, the basic algebra of a monocentric city is developed, and expressions derived for total number of properties $(N)^{1}$, total distance to properties $(\psi)$ and average distance to properties $(\varphi)$ in terms of density at the city centre $\left(d_{0}\right)$, the density gradient $(\lambda)$ and the radius of the urban area $(R)$. Varying these parameters enables a rich array of urban development scenarios to be generated. Attention is then focused on two such scenarios characterized as (a) Densification and (b) Suburbanization. Distribution cost elasticities for these cases are derived.

- Secondly, data on water distribution costs for 35 "urban districts" in the supply area of one of the water companies in England \& Wales is used to estimate the effect on these costs of variations in volume, numbers of properties and average distance to properties (measured as $\varphi$ ). Based on this relationship, distribution cost elasticities are quantified for each of the urban development scenarios, showing that in the case of densification, average distribution costs are decreasing in city size, whereas in the case of suburbanization these costs are increasing in city size.

- Next, the interaction with water production costs is considered, showing how, in the case of Densification, scale economies in production are reinforced by density economies in distribution, whereas in the case of $S u b$ urbanisation they are offset to a greater or lesser extent by diseconomies in distribution, i.e. higher spatial costs.

- A final section relates these findings to the literature on commuting costs, urban agglomeration and sprawl. It is concluded that high density settlement has the potential to reduce average costs in distribution (including commuting) as well as production, so that both favour agglomeration. Accordingly, urban modelers should be cautious about assuming that commuting (and other spatial costs) are always increasing in city size. 


\section{$2 \quad$ Modelling spatial costs in a monocentric city}

\subsection{Properties, density and distance}

An exact representation of the location of each and every property in a city is generally impractical ${ }^{2}$. For the purposes of this paper, urban areas are modeled as monocentric settlements with density falling away exponentially from the centre, which, in the majority of cases, is a reasonable approximation to the actual situation. This enables an expression for the average distance to properties $(\varphi)$ to be derived for each settlement, providing a compact summary measure of the spatial distribution of properties, which varies from place to place in line with its size and density gradient.

The basic algebra (and geometry) of the monocentric city can be summarized in a relationship between four parameters: $d_{0}$ (central density), $N$ (properties), $\lambda$ (density gradient) and $R$ (outer radius). Figure $\mathbf{1}$ is a bird's eye view of a monocentric city.

Figure 1. Monocentric city (top view)

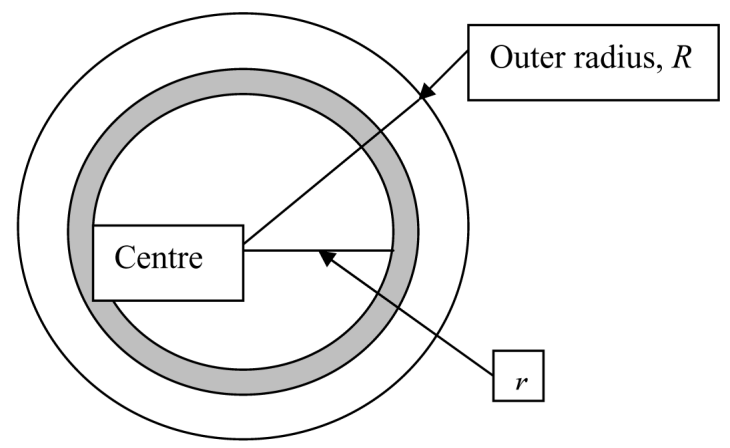

In Figure 1, if property density at radius $r$ is $d(r)$, then the total number of properties $(N)$ is given by:

$$
N=2 \pi \int_{0}^{R} d(r) \cdot r \cdot d r
$$

If, further, $d(r)=d_{0} \cdot e^{-\lambda r}$ (i.e. a negative exponential density gradient of $\lambda$ away from the centre, where density is $\left.d_{0}\right),(2)$ then gives:

$$
N=\frac{2 \pi d_{0}}{\lambda^{2}}\left[1-e^{-\lambda R}(1+\lambda R)\right]
$$


This is the basic relationship between $d_{0}$ (central density), $N$ (number of properties), $\lambda$ (density gradient) and $R$ (outer radius) and shows them to be interdependent - given any three, the fourth is fixed.

Further useful relationships concern the total and average distance of properties from the city centre. In Figure 1, the distance from the centre to a property in the shaded ring, where density is $d(r)$, is $r$, and so the total distance $(\psi)$ to all properties in the city is given by:

$$
\psi=2 \pi \int_{0}^{R} d(r) \cdot r^{2} \cdot d r=\frac{4 \pi \cdot d_{0}}{\lambda^{3}}\left[1-e^{-\lambda R}\left(1+\lambda R+\frac{\lambda^{2} R^{2}}{2}\right)\right]
$$

From (3) and (4), the average distance $(\varphi)$ from the centre to a property in the city is then given by:

$$
\varphi=\frac{\psi}{N}=\frac{2}{\lambda} \frac{\left[1-e^{-\lambda R}\left(1+\lambda R+\frac{\lambda^{2} R^{2}}{2}\right)\right]}{\left[1-e^{-\lambda R}(1+\lambda R)\right]}
$$

The implications of this expression are sketched in Figure 2 which shows a monocentric city in semi-profile and indicates how, for given $N$, higher values of $\lambda$ will be associated with a larger settlement radius $R$ if the central density $d_{0}$ is fixed.

Figure 2. Monocentric city (semi-profile) - Relationship between density and settlement radius for different values of the density gradient $\lambda$ (not to scale)

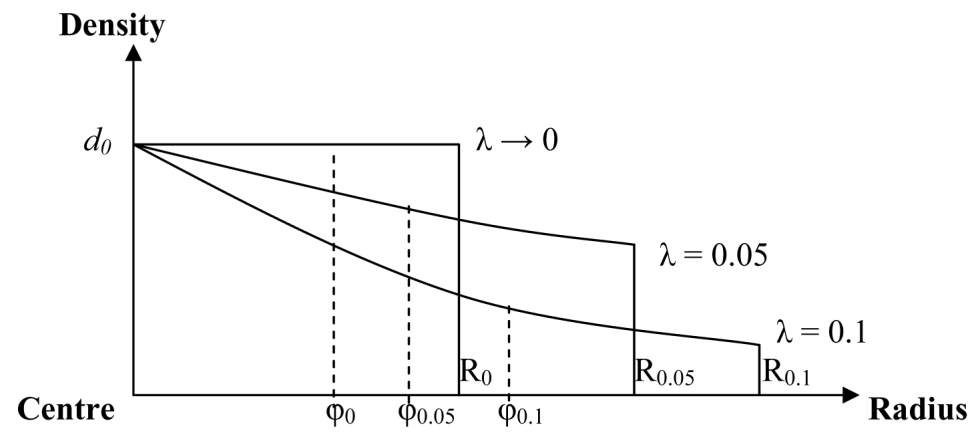

In Figure 2, the average distance to properties, $\varphi$, is indicated by the dotted lines: as the density gradient $\lambda \rightarrow 0, \varphi \rightarrow 2 / 3 R$; with higher values of $\lambda, \varphi$ increases as determined by $(4)^{3}$. 


\subsection{Urban development scenarios}

By varying the parameters in (3), a rich array of urban development scenarios can be generated. For example, even if $d_{0}$ is taken to be fixed, four interesting and contrasting cases can be obtained by varying one or more of $N, \lambda$ and $R$ :

a) Densification ${ }^{4}$ : Number of properties $(N)$ varies, while settlement radius $(R)$ is held constant (density gradient $\lambda$ also therefore varying);

b) Suburbanisation ${ }^{5}$ : Number of properties $(N)$ varies, holding $\lambda$ constant ( $R$ also therefore varying);

c) Dispersion: Density gradient $(\lambda)$ varies, holding number of properties $(N)$ constant ( $R$ also therefore varying);

d) Constant density: Number of properties $(N)$ varies, holding average density $(N / A)$ constant, where $A=\pi R^{2}$ (when both $\lambda$ and $R$ vary).

These cases cover a range of characteristics in the development of urban form that are likely to be of policy interest.

The resulting city configurations are portrayed in cross section in Figures 3 (a)-(d).

Figure 3(a). City cross-sections: $R$ constant, $N$ and $\lambda$ vary ('densification')

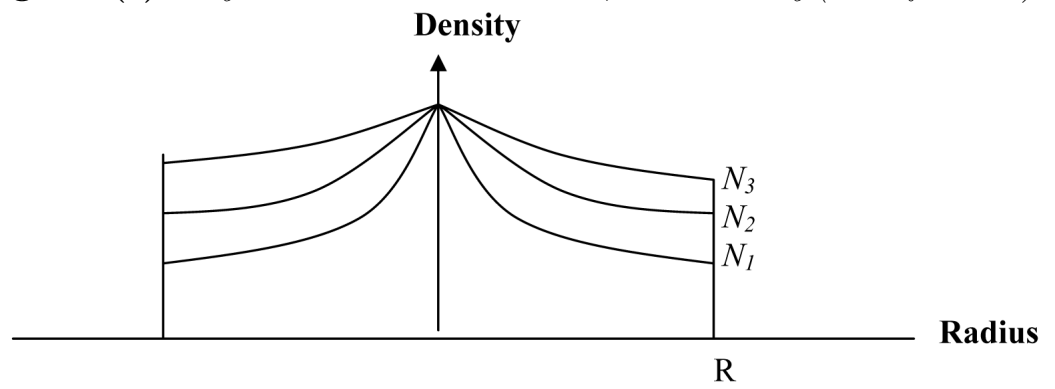

Figure 3(b). City cross-sections: $\lambda$ constant, $N$ and $R$ vary ('suburbanisation')

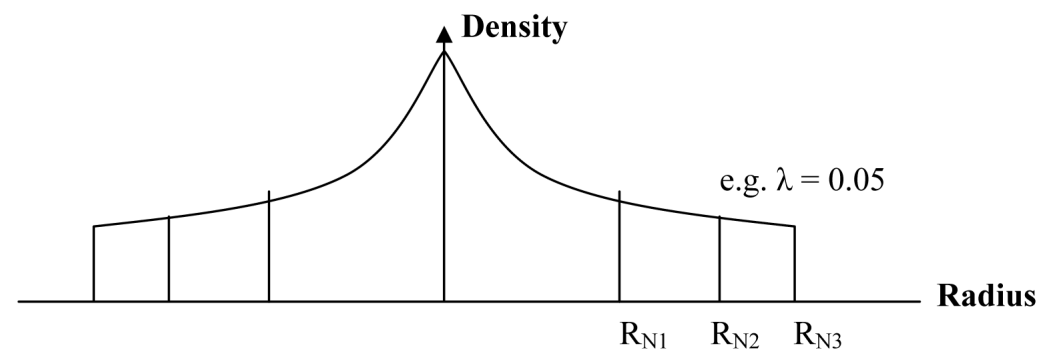
it is simply adopted as a convenient descriptive label.

5 The term "suburbanisation" is applied here to the case where the density gradient $(\lambda)$ does not change as the city expands, as this seems a good descriptor for what is portrayed in Figure 3(b). However, some authors have used changes in density gradient as a measure of suburbanization (e.g. Kopecky \& Suen (2009)). 
Figure 3(c). City cross-sections: $N$ constant, $\lambda$ and $R$ vary ('dispersion')

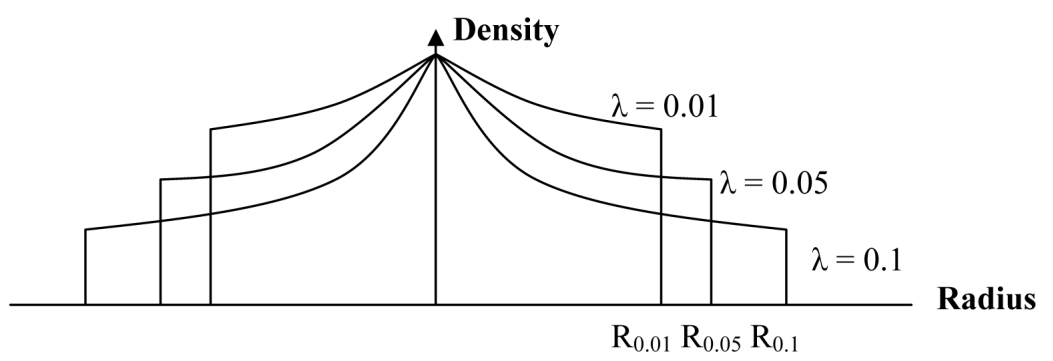

Figure 3(d). City cross-sections: Density constant, $N, \lambda$ and $R$ vary ('constant density')

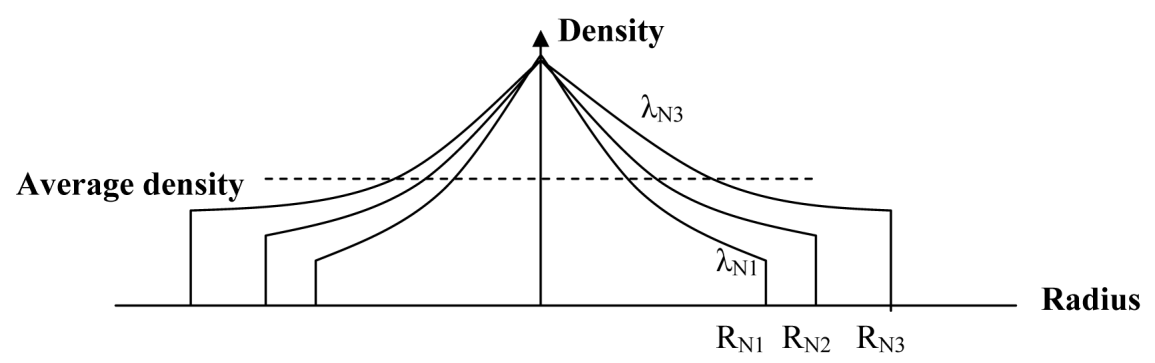

Of course, if $d_{0}$ is also allowed to vary, additional cases arise. For example, densification may take the form of an increase in density at the centre while $R$ is fixed; or dispersion may take the form of density falling at the centre as population moves outwards. To keep the discussion manageable, attention is here focused on just the two cases shown in Figure 3 (a) and $\mathbf{3}(\mathbf{b})$, i.e. densification and suburbanisation with $d_{0}$ fixed. This is sufficient to bring out the main points of interest.

\subsection{Distribution costs and elasticities}

In this paper, data on water distribution costs is used to illustrate the effect of urban form on spatial costs. The question how far the results can be generalized to a wider range of spatial costs (such as commuting) is taken up in Section 5 below. Following standard production theory, a cost function for water distribution would be derived from a production function for water distribution and would have the form:

$$
C D=f\left(D O, p_{v}, p_{k}, Z\right)
$$

Where $C D$ is distribution costs, $D O$ is distribution output, $p_{v}$ and $p_{k}$ are prices for variable and capital inputs respectively, and $Z$ is a vector of control variables (e.g. environmental factors). However, in relation to water distri- 
bution, it has become common to treat capital as 'quasi-fixed'(Garcia \& Thomas (2001), Torres \& Morrison Paul (2006)) so that (6) becomes a variable cost function with (quasi-fixed) capital $\bar{K}$ replacing $p_{k}$. Here, we take this a step further by assuming that the production function is of the Leontief type, allowing $\bar{K}$ to be dropped ${ }^{6}$. Furthermore, as our cost function will be applied to data for 35 "urban districts" all within the supply area of one company, it is reasonable to suppose that there will be little difference in $p_{v}$ between one area and another, so this term too can be dropped. As regards $Z$, the restricted domain of the data is again helpful but there could well be factors (such as differences in hilliness of terrain) which it would be desirable to control for. Unfortunately, information of this kind was not available but as our purpose here is only to present illustrative estimates in order to make some general points about distribution costs, we can still proceed.

That leaves $D O$ to consider. In utility studies, output is usually measured simply as the amount consumed, so missing the spatial aspect of the distribution stage. Here, which is an innovation in this context ${ }^{7}$, the output of the water distribution system $(D O)$ is measured as the product of the amount consumed $(Q C)$ and the average distance to properties $(\varphi)^{8} \cdot Q C$ in turn is the product of consumption per property $(w)^{9}$ and the number of properties $(N)$. Thus:

$$
D O=Q C . \varphi=w . N . \varphi
$$

However, it is not to be expected that each component of $D O$ will have the same impact on costs. Instead, a simple Cobb-Douglas form of the cost function has been adopted:

$$
\ln V C D=\alpha+\beta_{1} \ln w+\beta_{2} \ln N+\beta_{3} \ln \varphi
$$

Where $V C D$ is the variable costs of distribution.

Specification (8) provides an indication of the different effect on distribution costs of changes in volume, numbers of properties and average distance to properties. One elasticity, $\varepsilon_{w}$, falls out directly:

$$
\varepsilon_{w}=\frac{\partial(\ln V C D)}{\partial(\ln w)}=\beta_{1}
$$

6 Although there is some scope to vary the proportion of capital to other inputs in water distribution, in practice the network of pipes is more or less fixed and there is very little choice of technology so that significant change in input factor intensity is unlikely to be observed, particularly in a single year cross-section for one company. It is however common in transport studies to measure output using ton-miles, passenger-km, etc.

8 This implies that water is distributed from a central point whereas water treatment works are generally on the outskirts of towns. But if water is delivered in bulk to the distribution system, the effect on costs is not very material.

9 For simplicity, $w$ is taken to be uniform within each urban district in the subsequent analysis (although varying between districts). 
This can be viewed as a pure quantity effect, measuring the response of distribution costs to changes in water consumption per property, numbers of properties and other distribution area characteristics held constant. It does not vary with urban form.

However, the estimated coefficients $\beta_{2}$ and $\beta_{3}$ do not provide direct elasticity measures. This is because $N$ and $\varphi$ are both functions of $\lambda$ and $R$ and so are not independent of each other - see (3) and (5). One elasticity is of particular interest: $\varepsilon_{N}$, measuring the response of distribution costs to changes in the number of properties. To evaluate this elasticity, some algebraic manipulation is necessary and the value under densification is different from the value under suburbanization. In fact, as evaluation is easier (although still laborious) using $\psi$ rather than $\varphi$, a modification of (8) is adopted:

$$
\ln V C D=\alpha+\beta_{1} \ln w+\left(\beta_{2}-\beta_{3}\right) \ln N+\beta_{3} \ln \psi
$$

Now, under densification, $\mathrm{R}$ and $d_{0}$ are held constant as $N$ increases, with consequent change in $\lambda$ (and therefore $\psi$ ), and the required elasticity can be derived using (3) and (4) as follows ${ }^{10}$ :

$$
\begin{aligned}
& \varepsilon_{N / \bar{R}}=\left|\frac{\partial(\ln V C D)}{\partial(\ln N)}\right|_{d_{0}, \bar{R}}=\left(\beta_{2}-\beta_{3}\right)+\beta_{3}\left[\left|\frac{\partial(\ln \psi)}{\partial(\ln \lambda)}\right|_{d_{0}, \bar{R}} \cdot\left|\frac{\partial(\ln \lambda)}{\partial(\ln N)}\right|_{d_{0}, \bar{R}}\right] \\
& =\left(\beta_{2}-\beta_{3}\right)+\beta_{3}\left[\left|\frac{\lambda}{\psi} \frac{\partial \psi}{\partial \lambda}\right|_{d_{0}, \bar{R}} \cdot\left|\frac{N}{\lambda} \frac{\partial \lambda}{\partial N}\right|_{d_{0}, \bar{R}}\right] \\
& =\left(\beta_{2}-\beta_{3}\right)+\beta_{3}\left[\frac{3}{\lambda \varphi}\left[1-\frac{\lambda^{3} R^{3} e^{-\lambda R}}{6\left[1-e^{-\lambda R}\left(1+\lambda R+\frac{\lambda^{2} R^{2}}{2}\right)\right]}\right]\right)
\end{aligned}
$$

This is a somewhat complex expression but it may be noted that the term in square brackets depends only on $\lambda$ and $\bar{R}$. A value for $\varepsilon_{N / \bar{R}}<1$ would indicate increasing returns - costs increasing less than in proportion to number of properties - but at this stage it is not really possible to say more than that $\left(\beta_{2}-\beta_{3}\right)$ is likely to be negative while the term in $\beta_{3}$ will be positive.

Under suburbanization, $\lambda$ and $d_{0}$ are held constant as $N$ increases, with consequent change in $R$ (and therefore $\varphi$ ), and the required elasticity can be derived using (3) and (4) as follows ${ }^{10}$ :

10 Full derivation available on request from author. 


$$
\begin{aligned}
& \varepsilon_{N / \bar{\lambda}}=\left|\frac{\partial(\ln V C D)}{\partial(\ln N)}\right|_{d_{0}, \bar{\lambda}}=\left(\beta_{2}-\beta_{3}\right)+\beta_{3}\left[\left|\frac{\partial(\ln \psi)}{\partial(\ln R)}\right|_{d_{0}, \bar{\lambda}} \cdot\left|\frac{\partial(\ln R)}{\partial(\ln N)}\right|_{d_{0}, \bar{\lambda}}\right] \\
& =\left(\beta_{2}-\beta_{3}\right)+\beta_{3}\left[\left|\frac{R}{\psi} \frac{\partial \psi}{\partial R}\right|_{d_{0}, \bar{\lambda}} \cdot\left|\frac{N}{R} \frac{\partial R}{\partial N}\right|_{d_{0}, \bar{\lambda}}\right] \\
& =\left(\beta_{2}-\beta_{3}\right)+\beta_{3} \frac{R}{\varphi}
\end{aligned}
$$

This elasticity simplifies quite nicely and it depends only on $R$ and $\bar{\lambda}$. Since volume rises in line with $N$ (if $w$ is fixed), a value for $\varepsilon_{N / \bar{\lambda}}=1$ would indicate constant returns to scale. However, higher values are to be expected because of diseconomies associated with expansion into lower density suburbs.

\section{$3 \quad$ Estimated spatial costs and spatial elasticities under different urban development scenarios}

\subsection{Data used}

Information provided by one of the larger water companies in England \& Wales enabled me to put together data for 35 "urban districts", each comprising one urban area (as defined in ONS $(2004)^{11}$ ) together with its surrounding area of non-urban land. These cases therefore approximate monocentric cities. The main steps in processing of the data were as follows:

1) The company's information consisted of weekly records of numbers of properties, length of mains, daily water consumption and leakage for some 3000 District Metering Areas (DMAs). This was averaged and then aggregated by area, first for 184 Water Quality Zones (WQZs) ${ }^{12}$ and then for 55 "Urban Districts" ${ }^{13}$. Of these, 35 urban districts with only one large town (so omitting polycentric districts) were selected for analysis as providing a reasonable approximation to monocentric areas.

\footnotetext{
11 In ONS (2004) "urban areas" are defined as areas of built up land of at least $20 \mathrm{Ha}$, with a population of 1,500 or more.

12 DMAs are too small for our purposes, having little relationship to urban areas; WQZs are better but large urban areas may still comprise several WQZs, while in other cases more than one urban area is included in a WQZ.

13 The term "urban district" is adopted here as the areas concerned, being assembled from water company metering areas do not match standard administrative or statistical boundaries; rural areas without towns of more than 5000 population were omitted at this stage.
} 
2) This information was then matched with separate information on distribution operating costs for the same areas for the same year, similarly aggregated, providing the variable $V C D$.

3) Average consumption per property $(w)$ and number of properties $(N)$ comes from step 1 above. To obtain the other component of distribution output $(\varphi)$ for these urban districts, some simplifying assumptions are required:

- Each district can be treated as if it were a monocentric settlement;

- Next, a measure of area is needed. Actual areas (from step 1) include unoccupied or unserviced areas; but only areas having access to water mains can be serviced. The area of accessible land in each zone $\left(A_{\mathrm{o}}\right)$ was estimated as $M / 0.15$, where $M$ is length of mains. This is because $M / A$ is observed to be approximately 0.15 in fully urban zones; the argument then is that a similar ratio of mains to land with access to a supply will prevail in less urbanized zones (density of properties in terms of properties per $\mathrm{km}$ of mains is however generally much lower outside urban areas);

- Now the effective radius $(R)$ for each zone can be estimated as $R=\sqrt{A_{o} / \pi}$, where $A_{o}$ is the area of accessible land;

- The density gradient $\lambda$ can then be estimated from the observed property density $N / A_{\text {o }}$ by interpolation in a table which calculates density in properties/Ha for different values of $R$ and $\lambda$;

- Density at the centre of each zone $\left(d_{0}\right)$ is taken to be 30 properties/ $\mathrm{Ha}$ (a little above the highest value observed for any WQZ in the data);

- $\psi$ and $\varphi$ can then be calculated using (4) and (5).

Summary statistics for the resulting data set can be found in Appen$\operatorname{dix} \mathbf{A}$.

\subsection{Econometric estimates}

Implementing (10) for the 35 "urban districts" produced:

$$
\begin{gathered}
\ln V C D=-2.019+0.178 \ln w-0.729 \ln N+1.111 \ln \psi \\
\text { (S.E. 0.294) (S.E. 0.524) (S.E. 0.356) }
\end{gathered}
$$

From these results, $\beta_{1}=0.178, \beta_{2}=1.111-0.729=0.382$ and $\beta_{3}=$ 1.111 albeit with rather large standard errors for $\beta_{1}$ and $\beta_{2}$. $\beta_{1}$ provides an estimate of the elasticity of costs with respect to consumption per property, which (as might be expected) appears rather low, with a $10 \%$ increase raising costs by less than $2 \%$. To interpret $\beta_{2}$ and $\beta_{3}$, we need to make use of (11) and (12). 
From (11), the densification case:

$\varepsilon_{N / \bar{R}}=\left|\frac{\partial(\ln V C D)}{\partial(\ln N)}\right|_{d_{0}, \bar{R}}=\left(\beta_{2}-\beta_{3}\right)+\beta_{3}\left[\frac{3}{\lambda \varphi}\left(1-\frac{\lambda^{3} R^{3} e^{-\lambda R}}{6\left[1-e^{-\lambda R}\left(1+\lambda R+\frac{\lambda^{2} R^{2}}{2}\right)\right]}\right]\right]$

Taking $\beta_{2}=0.382$ and $\beta_{3}=1.111$ from (13), and using the 35 urban districts data, calculated values for this elasticity range from about 0.5 to about 0.8 , with a tendency for lower values of $\varepsilon_{N / \bar{R}}$ to be associated with lower values of $\lambda$ (See Figure 4 ). In all cases, this value is $<1$, indicating densification economies, which are stronger for relatively low $\lambda$ (higher density) ${ }^{14}$. So not only are there increasing returns but they increase as density increases. A possible economic interpretation is that higher densities allow larger bulk mains to be deployed with shorter runs between properties, so saving costs.

Figure 4. Relationship between densification elasticity $\mathcal{E}_{N / \bar{R}}$ and $\lambda$ for 35 "urban districts"

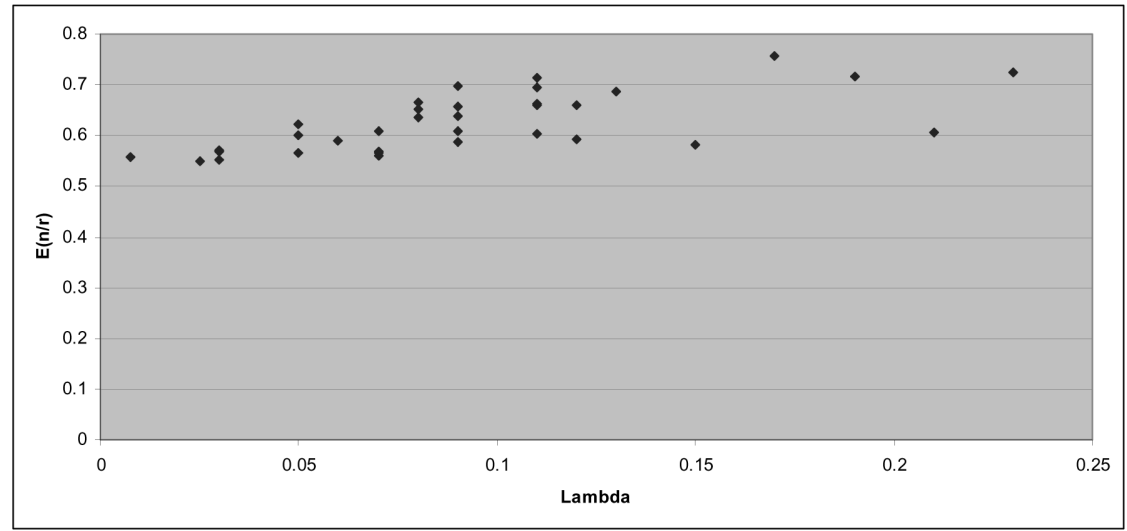

This can be contrasted with the suburbanization case.

$$
\text { From (12): } \varepsilon_{N / \bar{\lambda}}=\left(\beta_{2}-\beta_{3}\right)+\beta_{3} \cdot \frac{R}{\varphi}
$$


Taking again $\beta_{2}=0.382$ and $\beta_{3}=1.111$ from (13), and using the 35 urban districts data, calculated values for this elasticity range from about 1.03 to about 1.98 (See Figure 5). These values are all $>1$, indicating diseconomies as suburbanization proceeds, with higher values associated with higher values for $\lambda$ - i.e. the diseconomies intensify, the more rapidly density falls away from the centre (as might be expected). The economic interpretation is the flip side of the densification case: sparser settlement means smaller diameter pipes and longer runs between properties and hence higher unit distribution costs.

Figure 5. Relationship between suburbanization elasticity $\mathcal{E}_{N / \bar{\lambda}}$ and $\lambda$ for 35 "urban districts"

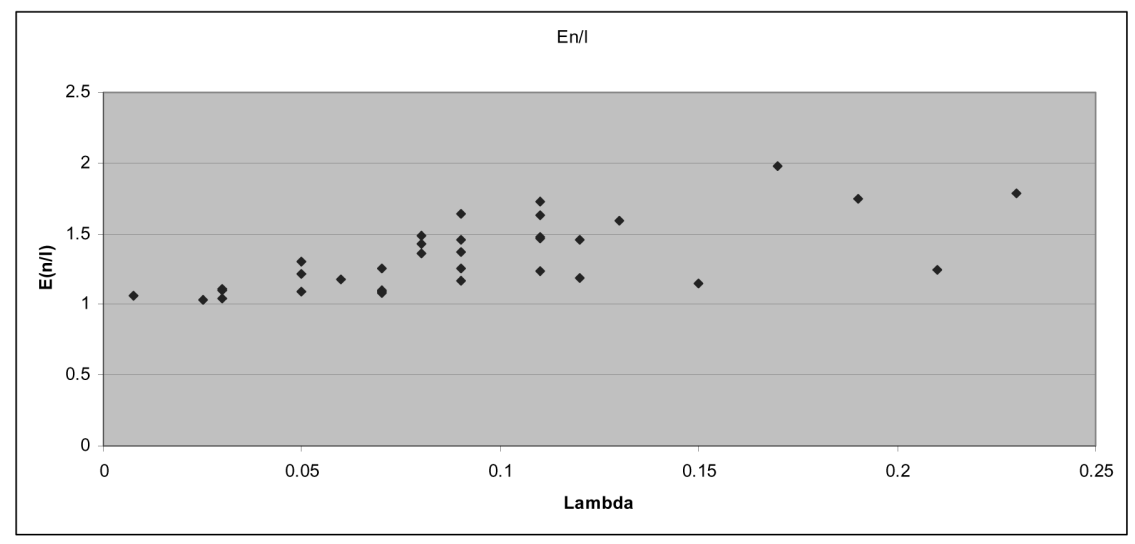

\subsection{Additional calculations}

The results portrayed in Figures $\mathbf{4}$ and $\mathbf{5}$ are for a rather mixed group of 35 urban areas, varying in size from a little over 2,000 properties to more than 600,000, and in (unadjusted) area from under 2,000 Ha to nearly $70,000 \mathrm{Ha}$. To further explore the implications for distribution costs of the densification and suburbanisation scenarios in Figure 3, illustrative calculations were carried out, starting with an average sized area, to show the effect on distribution costs of varying the number of properties $(N)$ either within a fixed radius (for densification) or with a fixed density gradient $(\lambda)$ (for suburbanization) ${ }^{15}$. In these calculations, estimated capital costs for distribution are also shown ${ }^{16}$. In summary, the calculations confirm that distribution costs depend strongly on the spatial configuration of the distri-

15 From the starting values for $N, \lambda$ and $R$, the method is to vary $N$ and use interpolation to infer either $\lambda$ or $R$ given the other variable, enabling a value for $\psi$ to be calculated. The estimated relationship (10) can then be used to obtain a value for $V C D$, taking an average value of 420 litres/property/day for $w$. 
bution area. With a monocentric structure, densification reduces unit distribution costs whereas greater suburbanisation raises them.

The results of the calculations for the 35 urban districts can be seen in Table $\mathbf{1}$ (for densification) and Table $\mathbf{2}$ (for suburbanization), expressed as unit costs as the implications are most easily appreciated in this form. The numbers to focus on are in the last 5 columns, where $V C D$ and $C C D$ are respectively the annual variable and capital costs of distribution, $U V C D$ and $U C C D$ are the related unit costs and $U T C D$ is the total unit cost. The 25,000 properties case is the base case in both tables. Table 1 then shows the effect on costs of increasing or decreasing the number of properties within a fixed radius (of $2.68 \mathrm{~km}$ ); while Table 2 shows the effect of increasing or decreasing the numbers of properties while holding the density gradient constant (at $0.06 \%$ per 100 metres).

Table 1 shows how adding properties within a fixed urban boundary substantially reduces unit distribution costs.

The more reliable figures are for variable costs, showing how this unit cost is more than halved from about $£ 163 / \mathrm{Ml}^{17}$ to about $£ 75 / \mathrm{Ml}$ as the number of properties rises from 5,000 to 50,000. The near constancy of the figure for capital costs $(C C D)$ may well be a consequence of allocating capital costs in proportion to length of mains but it is not unrealistic to suppose that the amount of pipework required to service a given area is not very sensitive to the number of properties served. If so, economies of scale will be very large, as shown by the figures for $U C C D$ and $U T C D$. This case thus provides a good illustration of density economies in distribution.

In contrast, Table 2 shows how adding properties with density gradient held constant has the opposite effect.

16 To obtain these estimated capital costs, the company total for capital maintenance plus return on capital was allocated to areas in proportion to length of mains. The estimated values are therefore very approximate and the results for capital costs should be viewed as indicative only; a possible bias is that there will be more large diameter pipes in dense, central areas, which will be undervalued by this method while the smaller pipes in less dense areas will be overvalued.

171 Megalitre $(\mathrm{MI})=1,000,000$ litres. 


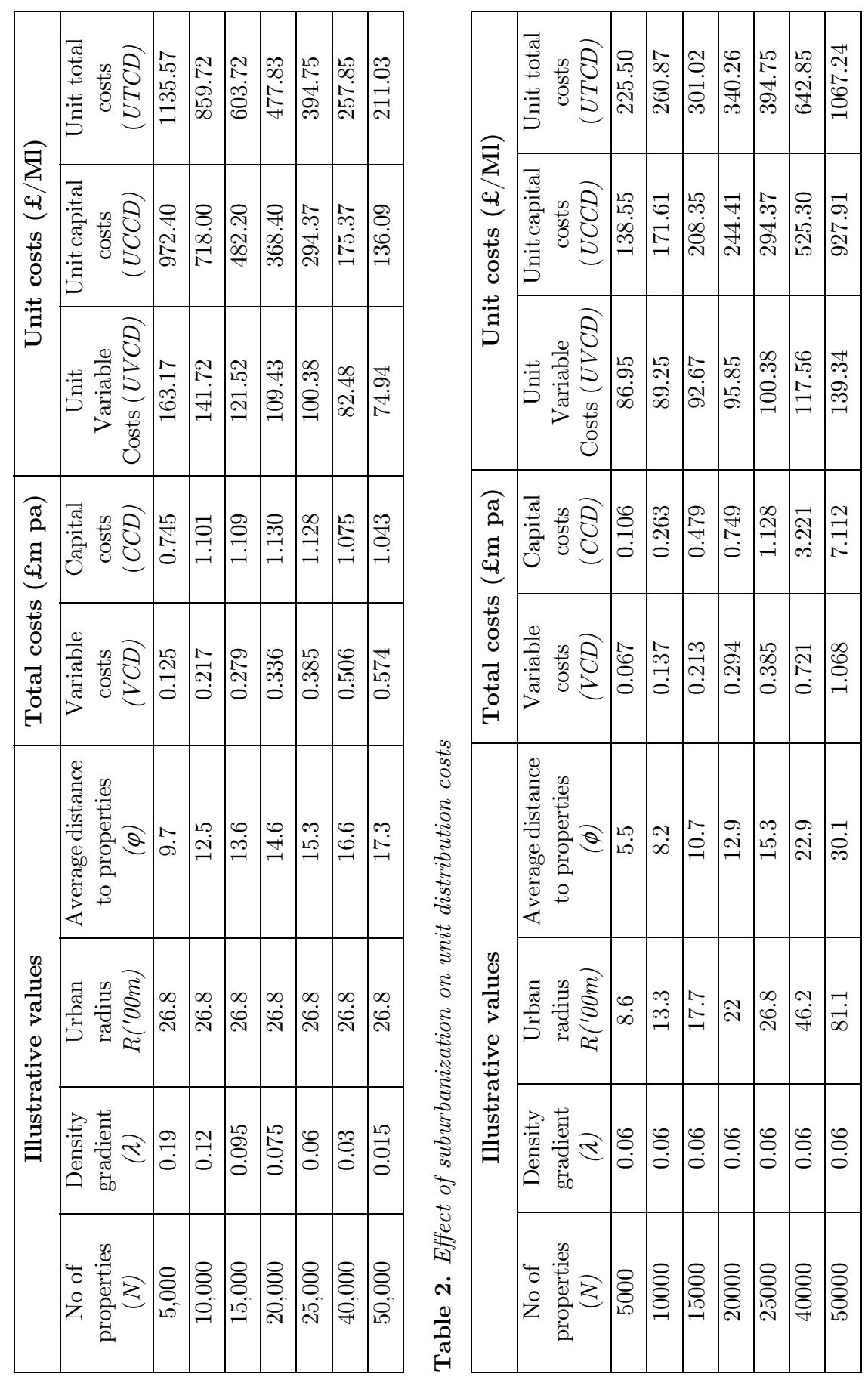


The effect on variable costs is relatively muted, with $U V C D$ rising from about $£ 87 / \mathrm{Ml}$ with 5,000 properties to nearly $£ 140 / \mathrm{Ml}$ with 50,000 properties. The estimated effect on capital costs is much more pronounced, which is not so surprising (despite caveats about the data) when it is noted that the urban radius increases from less than $1 \mathrm{~km}$ to over $8 \mathrm{~km}$ (and the average distance to properties rises from about $0.5 \mathrm{~km}$ to $3 \mathrm{~km}$ ). One way of viewing these suburbanization figures is as showing the effect of extending water supply from an urban core first to the suburbs and then to a rural fringe. The first 10,000 properties (the urban core) occupy only about 556 $\mathrm{Ha}$ at an average density of 18.0 properties/Ha. The next 15,000 properties (the suburbs) occupy about $1700 \mathrm{Ha}$ (average density 8.8 properties/Ha). The next 15,000 properties (the rural fringe) occupy about $4450 \mathrm{Ha}$ (average density 3.4 properties/Ha); and another 10,000 properties(bringing the total number of properties to 50,000) would add about 14,000 $\mathrm{Ha}$ at an average density of 0.7 properties/Ha. Compared with the total annual unit cost of distribution in the urban core, about $£ 261 / \mathrm{Ml}$, adding the suburbs raises this cost by about $50 \%$ to $£ 395 / \mathrm{Ml}$; adding the rural fringe adds another $60 \%$, bringing the cost to $£ 643 / \mathrm{Ml}$; and then with the outer fringe, the cost rises further to over $£ 1,000 / \mathrm{Ml}$, some five times the figure for the urban core alone. Clearly, the marginal cost of distribution to these more remote and highly dispersed properties is high ${ }^{18}$.

While not claiming great accuracy for these illustrative calculations, they are sufficiently grounded in reality to demonstrate that the effect on water distribution costs of urban expansion through densification are very different from those to be expected when expansion takes the form of suburbanization. The former case is characterized by scale economies whereas in the latter case there are scale diseconomies.

\section{$4 \quad$ Interaction of spatial costs with production economies}

The 35 "urban districts" were selected for analysis because they seemed to provide a reasonable approximation to the kind of monocentric settlement envisaged in our distribution model. Ideally, to assess the effect of bringing together water production and water distribution, one would use direct information about the relevant costs for each of the 35 districts. However, the water supply arrangements were found mostly not to be self-contained within these districts. Instead, to calculate water production costs, it is assumed that in each case water production is from a single water treatment

18 For the last 10,000 properties, the unit variable cost is $£ 227 / \mathrm{MI}$, some $250 \%$ higher than the $£ 89 / \mathrm{Ml}$ unit cost for the 10,000 properties in the urban core, while for unit capital costs the equivalent figures are $£ 172 / \mathrm{Ml}$ and £2538/MI. 
works (WTW) of the appropriate size, using the parameters obtained from another part of my research ${ }^{19}$. Illustrative cost calculations for hypothetical settlements of varying sizes and densities can then be carried out for the same two scenarios ("densification" and "suburbanization"), with distribution costs taken directly from Tables $\mathbf{1}$ and $\mathbf{2}$.

Thus, for water production, the average (or unit) cost $(£ / \mathrm{Ml})$ of production for a WTW producing $Q P \mathrm{Ml}$ day, based on data for 228 works operated by 21 companies in England \& Wales, is calculated as:

$$
U T C P=2^{0.31} \cdot 474 . Q P^{-0.24}
$$

If, in addition, for the purposes of these illustrative calculations, a leakage rate of $20 \%$ is assumed, then:

$$
Q P=Q C / 0.8
$$

The calculations in this section thus give a somewhat stylized view of the effect on production costs of different settlement characteristics. They do however help to show up such trade-offs as there are between economies of scale in production and diseconomies in distribution, without too many extraneous factors complicating the comparisons.

Now, the distribution costs shown in Table $\mathbf{1}$ can be brought together with production costs obtained using (14) to give illustrative total costs of water supply for the densification scenario, leading to the results shown in Table 3. In this table, TCP is the total cost of water production, TCD is the total cost of water distribution and $T C(P+D)$ is the total cost of water supply, comprising production and distribution. UTCP, UTCD and $U T C(P+D)$ are the related unit costs, obtained by dividing by $Q C$ converted to an annual rate (and assuming average consumption to be 420 litres/property/day).

Table 3 clearly shows the two-fold advantage of densification, leading to lower unit costs for both water production and water distribution. The unit cost of supply for a settlement of 50,000 properties is about one third of that for a settlement of 5,000 properties covering the same area.

As Table 4 shows, the suburbanisation case is more interesting: here the higher volumes produced as $N$ increases result in savings in unit water production costs, which fall by about $40 \%$ from $£ 583 / \mathrm{Ml}$ when $N=5,000$ to $£ 335 / \mathrm{Ml}$ when $N=50,000$, thus offsetting to some degree the increase in water distribution costs associated with serving less dense suburbs and rural areas. Total supply costs are now minimized at around 15,000 properties.

See Wenban-Smith (2009), Ch. IV. These parameters are for total production costs, including capital costs. 


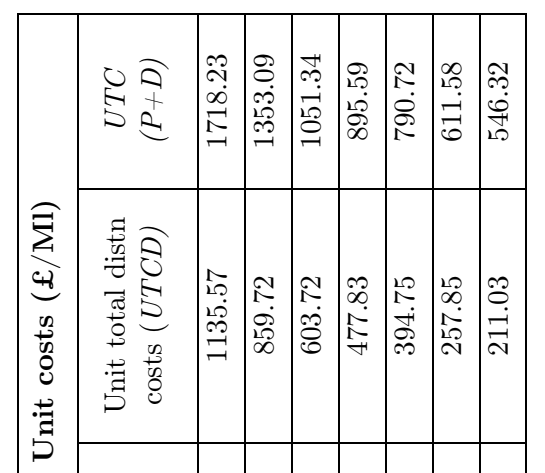

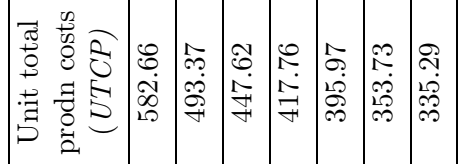

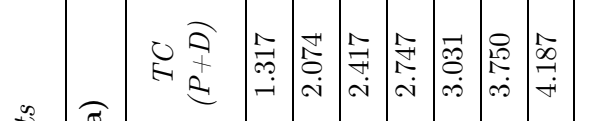

$\frac{5}{5}$

ก)

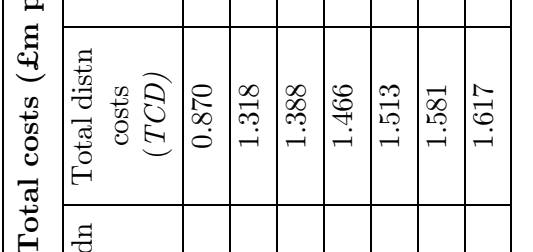

ह

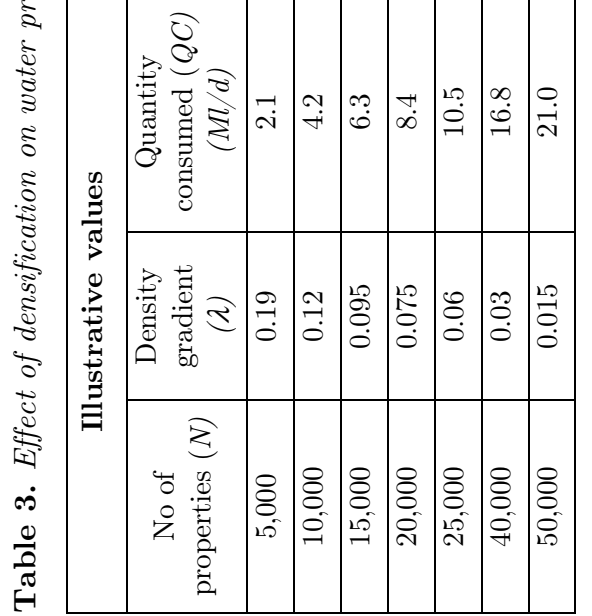

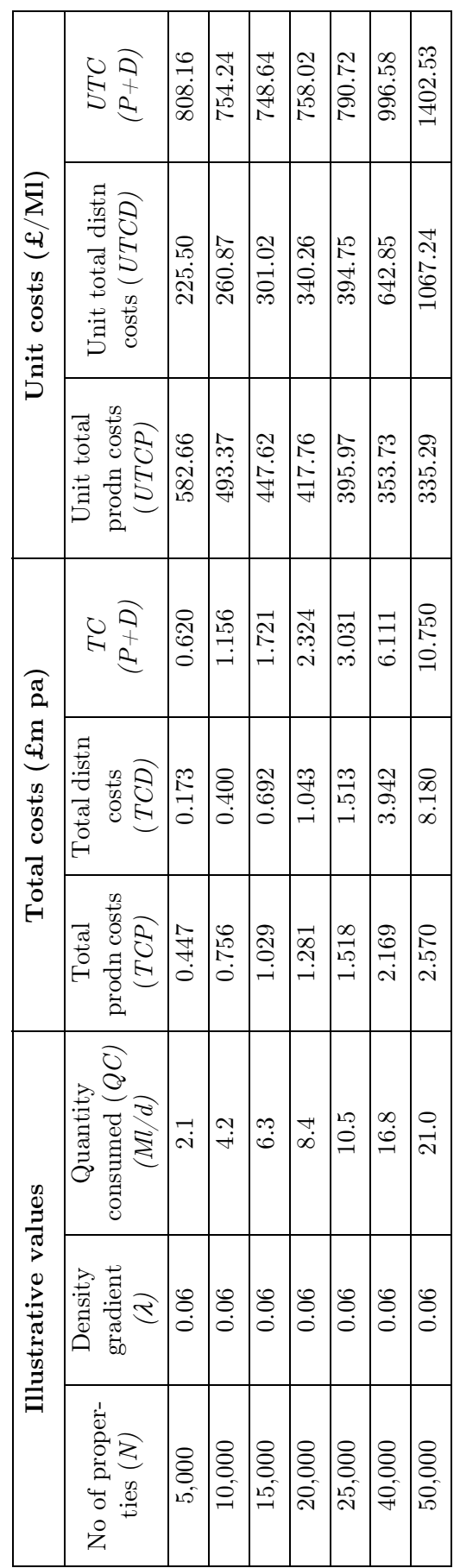


These results indicate that the benefits of more compact settlement can be substantial, as illustrated by the densification case. On the other hand, adding population by expanding into peripheral areas (suburbanization) introduces a trade-off between volume economies in production and diseconomies in distribution, which may on balance still be favourable, despite lower average density, at least for moderate expansion, as shown in Table 4.

\section{Conclusions and wider implications}

This examination of how water supply costs can be affected by urban form, although not claiming great precision, clearly shows that there is an important difference between the densification and suburbanisation cases. Only in the latter case are average distribution costs increasing in city size, like commuting costs in Arnott (1979), so introducing a trade-off between economies of scale in production and diseconomies in distribution. The question then is whether the water supply case provides a model that can be applied, mutatis mutandis, to wider range of urban costs with a spatial dimension.

Without carrying out further studies, it is only possible to offer some suggestive observations. Most of the relevant services can be seen as belonging to one of two broad types:

- Area-type: Services are provided within a defined area (e.g. water supply, other utilities, postal services, fire protection, transport systems). In such cases, getting the service to users involves distribution costs;

- Point-type: Services are provided at a specific point (e.g. hospitals, schools, offices, shops, museums, theatres, etc). In such cases, the equivalent consideration is the cost to users of accessing the facility.

Water supply, an example of the former type of service, was chosen for study because the technology is relatively simple and distribution costs are high so that the effects of interest should be particularly evident.

One characteristic of these kinds of services is that they require investment in infrastructure. Such infrastructure is generally regarded as being characterised by economies of scale. Thus McDonald (1997), discussing urbanisation economies in his standard text remarks (pp.40-41): "Economies of scale exist in the provision of inputs that are not specific to a particular industry. An important example is the general urban infrastructure." Similarly, Fujita (1989, p.135) observes that "... the provision of many public services and facilities (such as schools, hospitals, utilities, and highways) typically exhibits the characteristic of economies of scale." However, as the water supply case shows, there is an important difference in this regard between production and distribution. With production, economies of scale arise in the short run from fuller utilisation of existing works and in the long run from investment in larger works with lower unit costs; in the case of 
distribution, more intensive use of the existing network should also reduce unit costs but investment in extending the network to new areas may not do so, particularly if these areas are occupied at relatively low densities. Existing customers taking more water on average or more customers connecting to the existing system (provided its capacity is not exceeded) add little to distribution costs; indeed, the higher demand density may allow more economical systems (e.g. larger pipes) to be employed. But if new customers are located beyond the existing service boundary (as with suburbanisation), the impact on distribution costs can be substantial as the distribution system must then be extended, perhaps for the benefit of a relatively small number of customers.

It is not difficult to see that commuting costs share some of the same features. Bulking up of demand within an existing urban area enables roads to be more intensively used, and can make collective means of transport, such as buses and metros, more viable; on the other hand, if growth in population leads to expansion of the urban boundary, this will require new roads to be built while public means of transport are unlikely to become economic until the suburban population has increased considerably. However, transport also raises an additional issue: congestion, which is not a major consideration in the case of water supply ${ }^{20}$ but is of considerable importance in transport. Densification may be beneficial up to a point but risks increasingly higher costs beyond the point at which the capacity limits of transport systems are reached for at least some of the time. Such considerations lead to a more complicated story about the effect of urban form on transport costs. Arnott's expression for aggregate commuting costs - equation (1) can be adapted to the general monocentric city case by replacing the uniform density $\bar{d}$ with $d(r)=d_{0} \cdot e^{-\lambda r}$, and the unit transport cost $(t)$ can be modeled as declining as population increases (as better technologies are adopted), with implications similar to the water case. But $t$ may then increase (due to congestion) as density rises above some critical value. Only when the latter effect dominates will commuting costs play their accustomed role in urban models. Appendix B presents the results of some simple modeling to demonstrate these effects.

What about other types of infrastructure? It is likely that distribution costs are less significant in the case of electricity supply and telecommunications, although capital investment in distribution systems is still important. While in general lower distribution costs can be expected to favour agglomeration by extending the area that can be economically served, high capital costs nevertheless require that settlements be dense as well as relatively large if the necessary investments are to be viable ${ }^{21}$. At the same time, there have been some recent developments, such as small types of

20 The drop in pressure which can occur at times of peak demand for water is perhaps the nearest equivalent.

21 As the case of high capacity optical fibre cable perhaps demonstrates. 
sewage treatment works and local forms of power generation, which may help small settlements.

Application to Point-type infrastructure appears more straightforward. Taking the case of hospitals, for example, access costs, although often neglected, are relatively high while the extent of economies of scale in the production unit (i.e. the hospital) may only be modest. There would appear to be good potential to apply the methods developed here for water distribution costs to the costs of accessing hospitals (and other similar infrastructure), perhaps moderating enthusiasm for very large facilities.

In studies of agglomeration, it is common to use population as the measure of size ${ }^{22}$ and to suppose that commuting and other spatial costs are increasing in city size as so measured. One implication of the work reported here is that it may not be sufficient to look at numbers alone. Whereas increase in size through suburbanization would lead to diseconomies in distribution costs, increase in size through densification would, it seems, bring economies of scale (in water supply at least), with a positive influence on agglomeration. To get the full picture, it would appear necessary to give attention to density as well as size. It would be misleading, for example, to regard urban areas of similar size, as measured by population, as equivalent from an agglomeration perspective, if they have very different densities: lower density towns or cities are likely to have higher distribution costs. Put differently, agglomeration by densification would have real cost advantages (at least up to the point where congestion costs become appreciable ${ }^{23}$ ) whereas suburbanization would not. Accordingly, urban modelers should be cautious about assuming that commuting (and other spatial costs) are always increasing in city size.

\section{References}

Abdel-Rahman, H.M. \& A. Anas, (2004), "Theories of systems of cities" in Henderson J V \& J-F Thisse Handbook of Regional and Urban Economics, Volume 4 North-Holland

Arnott, R.J. (1979), "Optimal city size in a spatial economy" Journal of Urban Economics 6:65-89

Brueckner, J.K. (1982), "A note on sufficient conditions for negative exponential population densities" Journal of Regional Science 22, 353-359.

22 "The urban area population is the standard measure of urban size in studies of urbanisation economies." Eberts \& McMillen (1999, p.1481) Although urban areas will by definition probably have relatively high densities, there can still be considerable variation in density between one urban area and another.

23 While our data has not shown evidence of higher water distribution costs in larger, denser urban areas, such an effect does not seem a priori unlikely due to more difficult access, high rise buildings and higher wages. 
Eberts, R.W. \& D.P. McMillen, (1999), "Agglomeration economies and urban public infrastructure" in Cheshire P \& E S Mills (Eds) Handbook of Regional and Urban Economics, Vol 3 Elsevier North-Holland

Fujita, M. (1989), Urban Economics Cambridge University Press

Fujita, M. and J.-F. Thisse, (2002), Economics of Agglomeration: Cities, industrial location and regional growth Cambridge University Press

Garcia, S. and A. Thomas, (2001), "The structure of municipal water supply costs: Application to a panel of French local communities" Journal of Productivity Analysis, 16:5-29

Kopecky, K.A. and R.M.H. Suen, (2009), A quantitative analysis of suburbanization and the diffusion of the automobile, MPRA paper No. 13258, University of Munich.

McDonald, J.F. (1997), Fundamentals of Urban Economics Prentice Hall

ONS (2004) 2001 Census: Key statistics for urban areas in England 85 Wales, HMSO

Torres, M. and C.J. Morrison Paul (2006), "Driving forces for consolidation or fragmentation of the US water utility industry: A cost function approach with endogenous output" Journal of Urban Economics 59:104-120

Wenban-Smith, H.B. (2009), Economies of scale, distribution costs and density effects in urban water supply: A spatial analysis of the role of infrastructure in urban agglomeration $\mathrm{PhD}$ Thesis, London School of Economics.

\section{Appendix A: Summary of data for 35 urban districts}

\begin{tabular}{|l|c|c|c|c|}
\hline \multicolumn{1}{|c|}{ Variable } & Units & Average & Max & Min \\
\hline No of properties $(N)$ & Nos & 35,535 & 639,307 & 2,277 \\
\hline Household water consumption $(w)$ & Litres/prop/day & 423 & 738 & 335 \\
\hline $\begin{array}{l}\text { Urban district water consumption } \\
(Q C)\end{array}$ & Megalitres/day & 14.3 & 257.5 & 0.9 \\
\hline Gross area $(A)$ & Hectares & 20,550 & 123,988 & 1,173 \\
\hline Accessible area $\left(A_{0}\right)$ & Hectares & 2,826 & 35,336 & 211 \\
\hline Effective radius $(R)$ & 00 metres & 25.4 & 106.1 & 8.2 \\
\hline Average distance to properties $(\varphi)$ & 00 metres & 14.0 & 65.8 & 4.6 \\
\hline Density gradient $(\lambda)$ & $\%$ per `00m & 0.092 & 0.23 & 0.0075 \\
\hline Length of mains $(M)$ & km & 424 & 5,300 & 32 \\
\hline Distribution variable costs $(V C D)$ & $£ ’ 000$ & 548 & 9,930 & 41 \\
\hline Distribution capital costs $(C C D)$ & $£ ’ 000$ & 1,347 & 16,839 & 100 \\
\hline
\end{tabular}




\section{Appendix B: Illustrative calculations of effect of urban form on commuting costs}

In this Appendix, calculations are presented to illustrate the effect on average distance to properties $(\varphi)$ and average commuting cost $(t . \varphi)$ of three urban growth cases: (a) Densification; (b) Suburbanisation; and (c) Arnotttype (uniform density). In the first two cases, the first four columns of the tables below are taken directly from the first four columns of main text Tables 1 and $\mathbf{2}$ respectively. For the Arnott-type case, $\lambda$ is effectively zero and $\varphi=2 / 3 R$.

For commuting cost, it is supposed that commuting cost per unit distance $(t)$ is decreasing in $N$ (due to economies of scale in public transport) but increasing in density above some critical value $\bar{d}$ (due to congestion). For the purpose of the calculations, the specification adopted is:

$$
t=1-0.00001 N+0.01(d-\bar{d})^{2}
$$

It should be obvious that this specification has no empirical basis and is adopted solely for illustrative purposes. In the same spirit, it is assumed that $\bar{d}=15$ properties $/$ Ha.

These assumptions lead to the results shown in Tables B1, B2 and B3:

Table B1. Densification - Illustrative calculations of $\varphi$ and commuting cost

\begin{tabular}{|c|c|c|c|c|c|}
\hline $\mathbf{N}$ & $\boldsymbol{\lambda}$ & $\mathbf{R}$ & $\boldsymbol{\varphi}$ & $\mathbf{t}$ & $\mathbf{t . \varphi}$ \\
\hline 5000 & 0.19 & 26.8 & 9.7 & 0.95 & 9.17 \\
\hline 10000 & 0.12 & 26.8 & 12.5 & 0.90 & 11.25 \\
\hline 15000 & 0.095 & 26.8 & 13.6 & 0.85 & 11.60 \\
\hline 20000 & 0.075 & 26.8 & 14.6 & 0.80 & 11.66 \\
\hline 25000 & 0.06 & 26.8 & 15.3 & 0.75 & 11.45 \\
\hline 40000 & 0.03 & 26.8 & 16.6 & 0.67 & 11.20 \\
\hline 50000 & 0.015 & 26.8 & 17.3 & 1.01 & 17.47 \\
\hline
\end{tabular}


Table B2. Suburbanisation - Illustrative calculations of $\varphi$ and commuting cost

\begin{tabular}{|c|c|c|c|c|c|}
\hline $\mathbf{N}$ & $\boldsymbol{\lambda}$ & $\mathbf{R}$ & $\boldsymbol{\varphi}$ & $\mathbf{t}$ & $\mathbf{t . \varphi}$ \\
\hline 5000 & 0.06 & 8.6 & 5.5 & 1.37 & 7.53 \\
\hline 10000 & 0.06 & 13.3 & 8.2 & 0.99 & 8.16 \\
\hline 15000 & 0.06 & 17.7 & 10.7 & 0.85 & 9.09 \\
\hline 20000 & 0.06 & 22 & 12.9 & 0.80 & 10.35 \\
\hline 25000 & 0.06 & 26.8 & 15.3 & 0.75 & 11.45 \\
\hline 40000 & 0.06 & 46.2 & 22.9 & 0.60 & 13.71 \\
\hline 50000 & 0.06 & 81.1 & 30.1 & 0.50 & 15.07 \\
\hline
\end{tabular}

Table B3. Arnott-type (uniform density) - Illustrative calculations of $\varphi$ and commuting cost

\begin{tabular}{|c|c|c|c|c|c|}
\hline $\mathbf{N}$ & $\boldsymbol{\lambda}$ & $\mathbf{R}$ & $\boldsymbol{\varphi}$ & $\mathbf{t}$ & $\mathbf{t . \varphi}$ \\
\hline 5000 & 0 & 12.0 & 8.0 & 0.95 & 7.58 \\
\hline 10000 & 0 & 16.9 & 11.3 & 0.90 & 10.16 \\
\hline 15000 & 0 & 20.7 & 13.8 & 0.85 & 11.75 \\
\hline 20000 & 0 & 23.9 & 16.0 & 0.80 & 12.77 \\
\hline 25000 & 0 & 26.8 & 17.9 & 0.75 & 13.39 \\
\hline 40000 & 0 & 33.9 & 22.6 & 0.60 & 13.55 \\
\hline 50000 & 0 & 37.9 & 25.2 & 0.50 & 12.62 \\
\hline
\end{tabular}

The implications are more easily appreciated by looking at Figures B1 and B2. In Figure B1, average distance to properties $(\varphi)$ is plotted. It can be seen to be increasing in $N$ in all three scenarios: most steeply with suburbanization; least steeply with densification; while theArnott case is intermediate.

Figure B1. Average distance to properties ( $\varphi$ ) under densification, suburbanization and Arnott-type cases

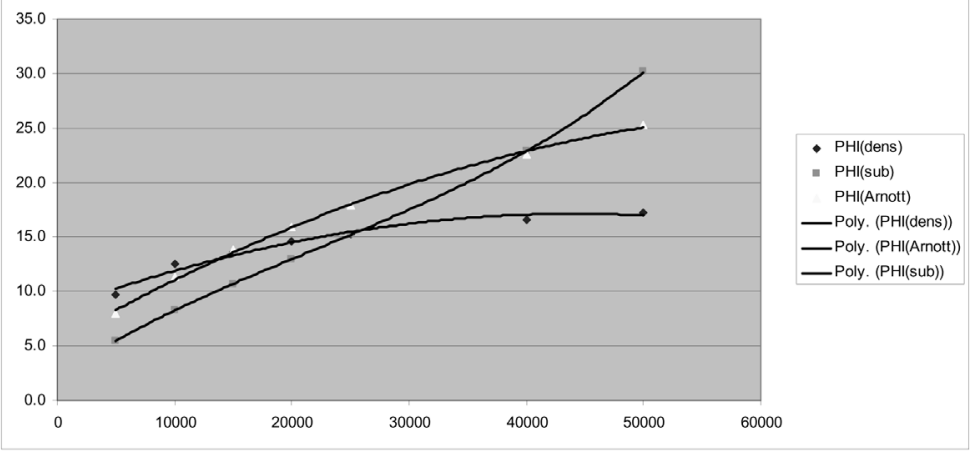


In Figure B2, average commuting cost is plotted. This is $\varphi$ multiplied by the unit transport cost $t$, calculated using (B1) above. This is more interesting. With suburbanization, average commuting cost rises steadily, the increase in average distance outweighing economies of scale in transport. With the Arnott case, average distance increases less fast and economies of scale predominate above about 30,000 properties. With densification, this effect is more marked with economies of scale predominating above about 15,000 properties but then congestion kicks in reversing the trend. The implication for urban modelling is that commuting costs will only play their accustomed role as a negative influence on agglomeration over the rising portions of these curves. Where the curve is downward sloping, the influence of commuting costs on agglomeration will be positive. Of course, these conclusions only follow if our calculations provide a reasonable representation of such costs. That is a matter for empirical investigation. But urban modelers should be cautious about assuming that commuting (and other similar spatial costs) are always increasing in city size as measured by numbers of properties or population.

Figure B. Average commuting cost (t. $\varphi$ ) under densification, suburbanization and Arnott-type cases

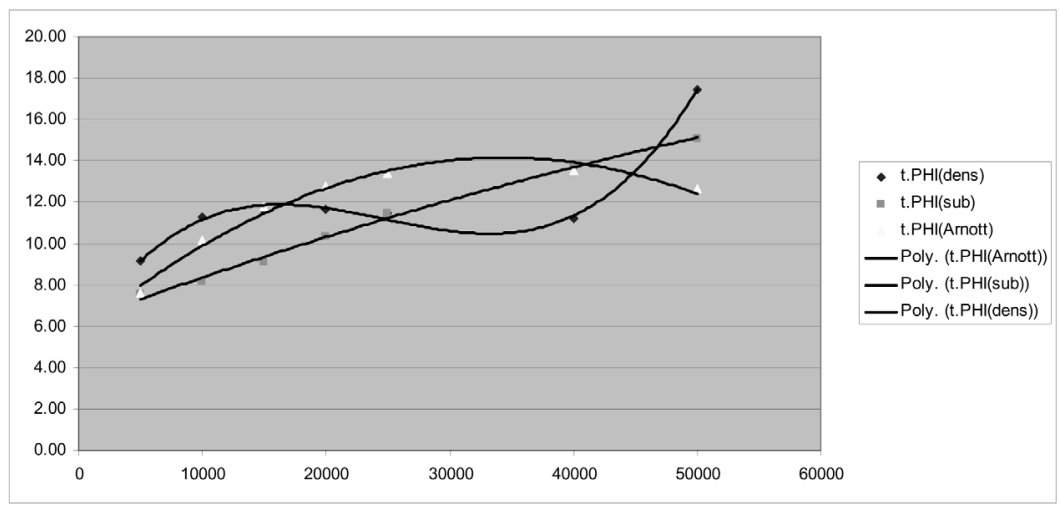

